\title{
The ATLAS Measurements of Jet Production and the Strong Coupling Constant
}

\author{
Lee Sawyer* \\ On behalf of the ATLAS Collaboration \\ Louisiana Tech University \\ E-mail: sawyeralatech.edu
}

\begin{abstract}
The production of jets at hadron colliders provides a stringent test of perturbative QCD at the highest energies. The process can also be used to probe the gluon density in the parton distribution function of the proton. Specific topologies can be used to extract the strong coupling constant. The ATLAS collaboration has recently measured the inclusive jet production cross section in data collected at a center-of-mass energy of $8 \mathrm{TeV}$ and $13 \mathrm{TeV}$ at the Large Hadron Collider (LHC). The measurements have been performed differentially in jet rapidity and transverse momentum. The collaboration also presents a first measurement of the dijet cross section at a center-of-mass energy of $13 \mathrm{TeV}$ as a function of the dijet invariant mass and rapidity. The results have been compared with state-of-the-art theory predictions at NLO in pQCD, interfaced with different parton distribution functions and can be used to constrain the proton structure. We also present new measurements of transverse energy-energy correlations (TEEC) and their associated asymmetries (ATEEC) in multi-jet events at a center-of-mass energy of $8 \mathrm{TeV}$. The data is unfolded to the particle level and compared to the expectations from parton shower Monte Carlo programs as well as from NLO pQCD calculations. The latter are also used to extract the strong coupling constant and test the renormalization group equation.
\end{abstract}

The European Physical Society Conference on High Energy Physics

5-12 July, 2017

Venice

${ }^{*}$ Speaker. 
The production of collimated jets of particles from the fragmentation and hardonization of quarks and gluons is the leading inelastic process at hadron colliders. The study of the production of jets is an important test of the Standard Model, and is a necessary prerequisite in understanding backgrounds to many other measurements and searches. The Large Hadron Collider (LHC) allows an unprecedented reach in jet energies, while state-of-the-art theoretical predictions in the framework of perturbative Quantum Chromodynamics (pQCD) allow next-to-leading order (NLO) comparisons of 3- and 4-jet final states, and next-to-next-to-leading order (NNLO) comparisons to inclusive jet and dijet production. These measurements also provide important input into Parton Distribution Functions (PDFs) and the tuning of Monte Carlo generators. Jet final states can also be studied for evidence of new strongly produced particle states.

In this contribution, we present recent results on inclusive jet and dijet measurements made with the ATLAS [1] experiment at the LHC, along with our determination of the strong coupling constant $\alpha_{s}$ based transverse energy-energy correlations and their asymmetries.

\section{Inclusive Jets}

Inclusive jet production has been previously measurement by ATLAS at $\sqrt{s}=7 \mathrm{TeV}$ [2]. Recent results are now presented for inclusive jet production at $8 \mathrm{TeV}$ and at $13 \mathrm{TeV}$. In Fig. 1, we show the $8 \mathrm{TeV}$ result, based on $20.2 \mathrm{fb}^{-1}$ of data collected. Jets are reconstructed with the anti- $k_{t}$ jet algorithm, with radius parameter $R=0.4$ or 0.6 , and are required to have $p_{T}>70 \mathrm{GeV}$ and be within a rapidity of $|y|<3.0$. The measurement is made doubly differential in jet $p_{T}$ and rapidity. The data are compared to the NLO pQCD prediction with the MMHT2014 PDF set corrected for non-perturbative and electroweak effects. The error bars indicate the statistical uncertainty and the systematic uncertainty in the measurement added in quadrature. The statistical uncertainty is shown separately by the inner vertical line. The reader is referred to the paper [3] for full description of data/theory comparisons and comparisons with various PDF sets, including detailed $\chi^{2}$ tests.

Similar measurements are also made of inclusive jet production at $\sqrt{s}=13 \mathrm{TeV}$, based on 3.2 $\mathrm{fb}^{-1}$ of data. Jets reconstructed with the anti- $k_{t}$ algorithm with $R=0.4$ are accepted if they have $p_{T}>100 \mathrm{GeV}$ within $|y|<3$. Results showing the comparison of data to NLO pQCD prediction are shown in Fig. 2 (left), with different PDF sets as noted. Further comparisons, including detailed $\chi^{2}$ tests, can be found in [4]. For the first time, comparisons are also made to NNLO pQCD predictions [5] for inclusive jet production. Comparisons of NNLO to NLO pQCD as a function of jet $p_{T}$ are shown in Fig. 2 (right).

\section{Dijets}

At this conference we have presented the first measurement of dijet production cross sections at $13 \mathrm{TeV}$. Events were required to have at least two $R=0.4$ anti-kt jets with $p_{T}>75 \mathrm{GeV}$ within $|y|<$ 3 , and the leading two jets were also required to have $H_{T}=p_{T 1}+p_{T 2}>200 \mathrm{GeV}$ in order to reduce instabilities in the corresponding NLO theory calculations. A double differential measurement is made in the invariant mass $M_{j j}$ of the two leading jets and in $y^{*}=\left|y_{1}-y_{2}\right| / 2$. Results are shown Fig. 3 for various PDF sets. Additional comparisons including detailed $\chi^{2}$ tests can be found in Ref. [4]. 

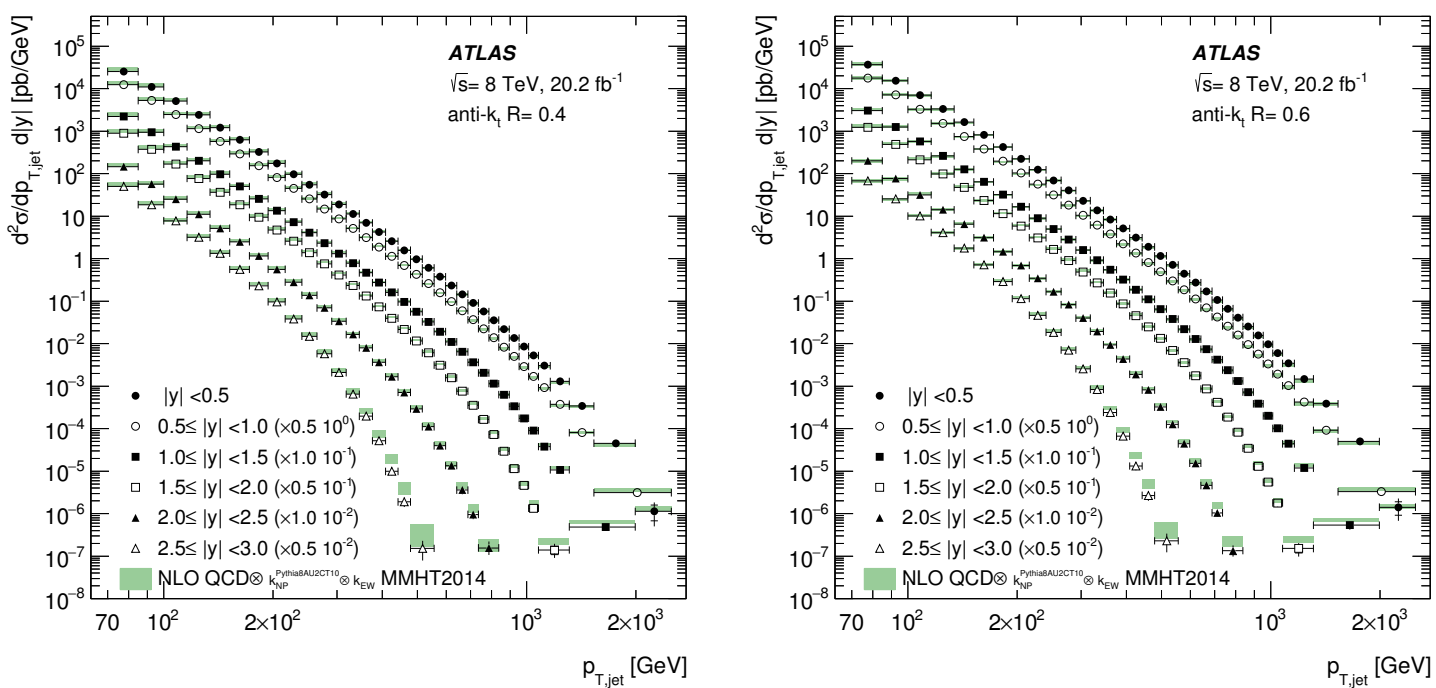

Figure 1: The inclusive jet cross-section at $8 \mathrm{TeV}$ as a function of jet $p_{T}$ in bins of jet rapidity [3]. The results are shown for jets identified using the anti- $k_{t}$ algorithm with $R=0.4$ (left) and $R=0.6$ (right). For better visibility the measurements are multiplied by the factors indicated in the legend.
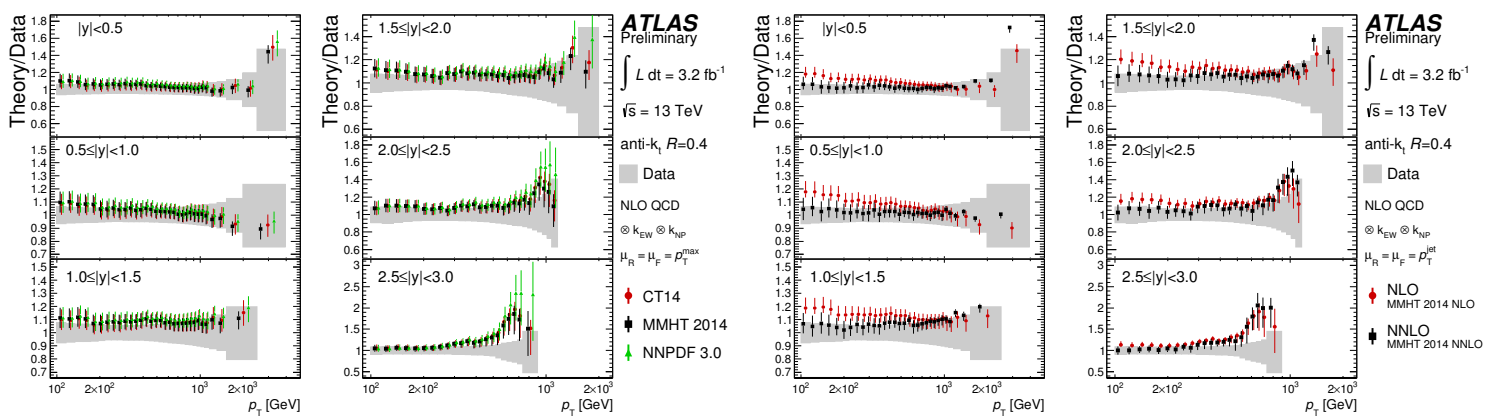

Figure 2: a) Comparison of the measured inclusive jet cross-section at $13 \mathrm{TeV}$ and the NLO pQCD predictions [4]. The ratios of predictions and data are shown as a function of the jet $p_{T}$ in six $|y|$ bins for anti- $k_{t}$ jets with $R=0.4$. The predictions calculated using NLOJET++ with different PDF sets (CT14, MMHT 2014, NNPDF 3.0) are shown. b) Ratios of the NLO and NNLO pQCD predictions to the measured inclusive jet cross-sections are shown as a function of the jet $p_{T}$ in six $|y|$ bins for anti-kt jets with $R=0.4$. The NLO predictions are calculated using NLOJET++ with the MMHT 2014 NLO PDF set. The NNLO predictions are provided by the authors of Ref. [5] using NNLOJET with $p_{T}^{\text {jet }}$ as the QCD scale and the MMHT 2014 NNLO PDF set.

\section{Energy-energy Correlations and $\alpha_{s}$}

Energy-energy correlations are energy-weighted angular distributions. The transverse energyenergy correlations (TEEC) are weighted by $x_{T i}=E_{T i} / E_{T}$ where $E_{T}=\sum E_{T i}$ is the scalar sum of the transverse energies of the jets in an event. The measured quantity is then defined as

$$
\frac{1}{\sigma} \frac{d \Sigma}{d \cos \phi}=\frac{1}{\sigma} \sum_{i j} \frac{d \sigma}{d x_{T i} d x_{T j} d \cos \phi} x_{T i} x_{T j} d x_{T i} d x_{T j}
$$



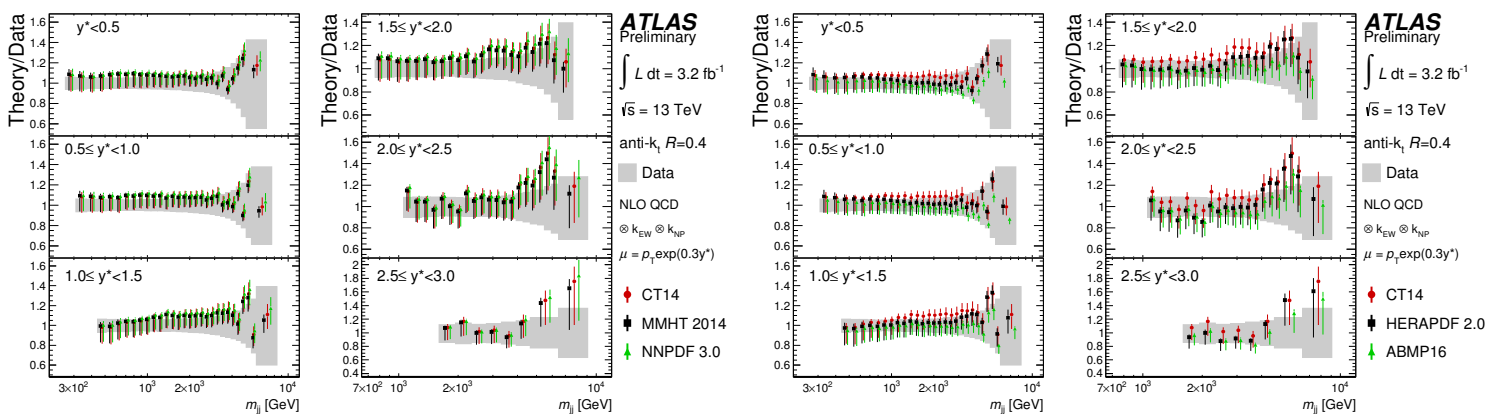

Figure 3: Comparison of the measured dijet cross-sections and the NLO pQCD predictions shown as the ratios of predictions to the measured cross-sections [4]. These ratios are shown as a function of the dijet $M_{j j}$ in six $y^{*}$ bins for anti-kt jets with $R=0.4$. The predictions calculated using NLOJET++ with different PDF sets are shown.

TEEC have been calculated at order $\alpha_{s}^{2}$ in $\mathrm{pQCD}$ and can thus be used to make a determination of $\alpha_{s}$. By measuring the TEEC asymmetry (ATEEC)

$$
\frac{1}{\sigma} \frac{d \Sigma^{\mathrm{asym}}}{d \cos \phi}=\left.\frac{1}{\sigma} \frac{d \Sigma}{d \cos \phi}\right|_{\phi}-\left.\frac{1}{\sigma} \frac{d \Sigma}{d \cos \phi}\right|_{\pi-\phi}
$$

effects which are uniform in azimuthal angle $\phi$ cancel.

ATLAS first presented a measurement of TEEC and ATTEC based on data collected at $\sqrt{s}=$ $7 \mathrm{TeV}$ [6], which yielded a value of $\alpha_{s}\left(M_{Z}\right)=0.1172 \pm 0.0010$ (exp. $)_{-0.0026}^{+0.0065}$ (theor.). At this conference, we present the first measurement of TEEC and ATEEC at $13 \mathrm{TeV}$ [7]. The new results are based on $20.2 \mathrm{fb}^{-1}$ of data collected at $\sqrt{s}=8 \mathrm{TeV}$. Events were required to have at least two jets with $p_{T}>100 \mathrm{GeV}$ within $|y|<2.5$, and $H_{T 2}=p_{T 1}+p_{T 2}>800 \mathrm{GeV}$. Events were binned differentially in bins of $\cos \phi$ and $H_{T 2}$.

Since the TEEC are essentially ratios of three-jet to two-jet cross sections, the NLO predictions for these cross sections can be fit to the experimental distributions varying $\alpha_{s}$. The fitted value of $\alpha_{s}$ can then be related to $\alpha_{s}\left(M_{Z}\right)$ using the two-loop renormalization group equation. Fits are taken in each $H_{T 2}$ bin. The results for TEEC (left) and ATEEC (right) are shown Fig. 4, along with comparisons to NLO pQCD, for the bin of $800<H_{T 2}<850 \mathrm{GeV}$; see the paper for similar distributions in the other $H_{T 2}$ bins and details of the fitting procedure.

The values of $\alpha_{s}$ determined from the fits above are shown in Fig. 5, along with results from other experiments and the previous world average. The global fitted value for $\alpha_{s}$ extrapolated to $M_{Z}$ is $\alpha_{s}\left(M_{Z}\right)=0.1162 \pm 0.0011(\text { exp. })_{-0.0061}^{+0.0076}($ scale $) \pm 0.0018(\mathrm{PDF}) \pm 0.0003(\mathrm{NP})$ for the TEEC measurement, and $\alpha_{s}\left(M_{Z}\right)=0.1196 \pm 0.0013(\text { exp. })_{-0.0013}^{+0.0061}($ scale $) \pm 0.0017(\mathrm{PDF}) \pm 0.0004(\mathrm{NP})$ for the ATEEC measurement, where the systematic errors are separated into those due to variation of the renormalization and factorization scales ("scale"), the variations of the PDFs ("PDF"), and the uncertainties due to non-perturbative corrections ("NP"). Both values are in good agreement with the world average. 

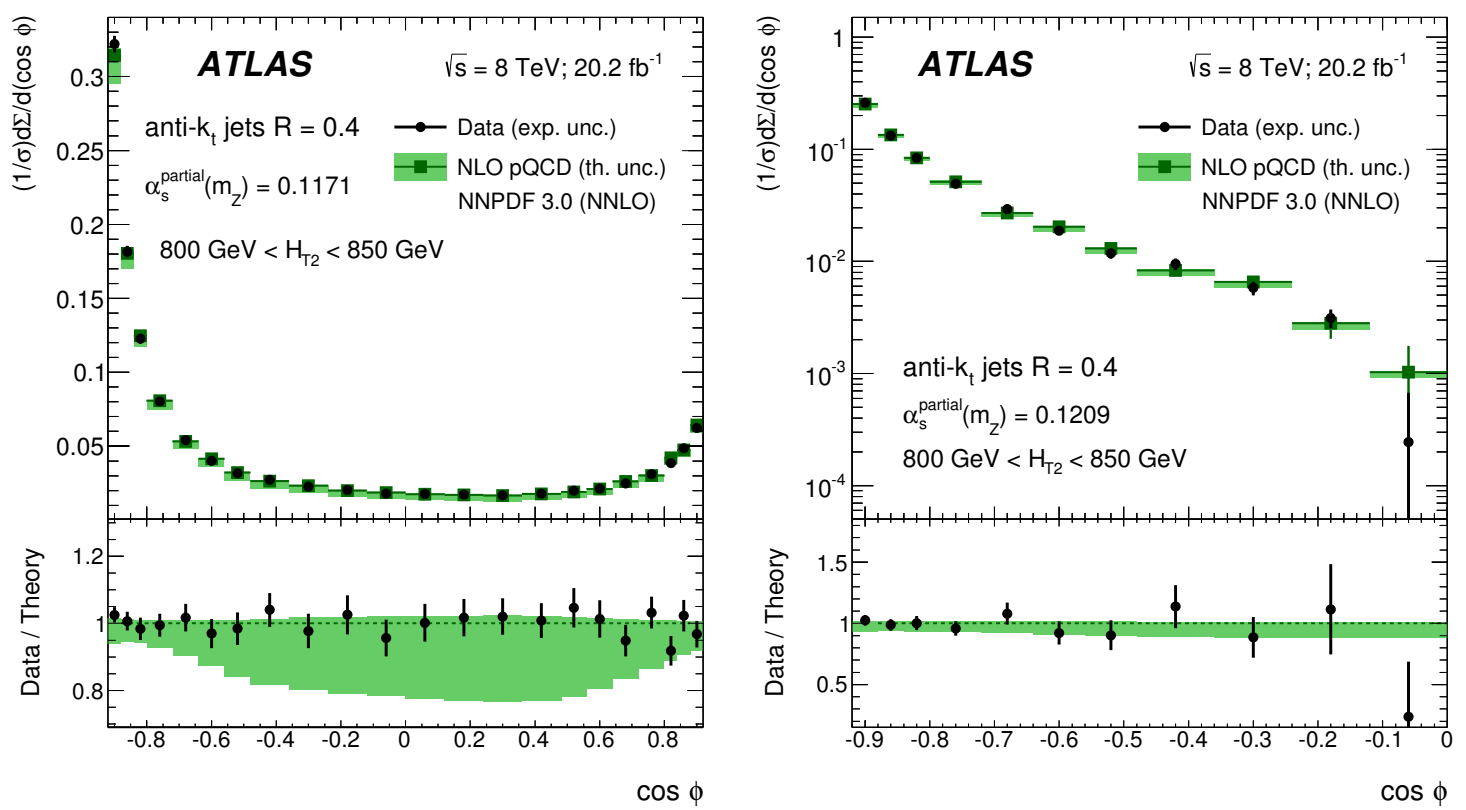

Figure 4: Comparison of the TEEC (left) and ATEEC (right) data and the theoretical predictions after the fit. The value of $\alpha_{S}\left(M_{Z}\right)$ used in this comparison is fitted independently for each energy bin. [7]
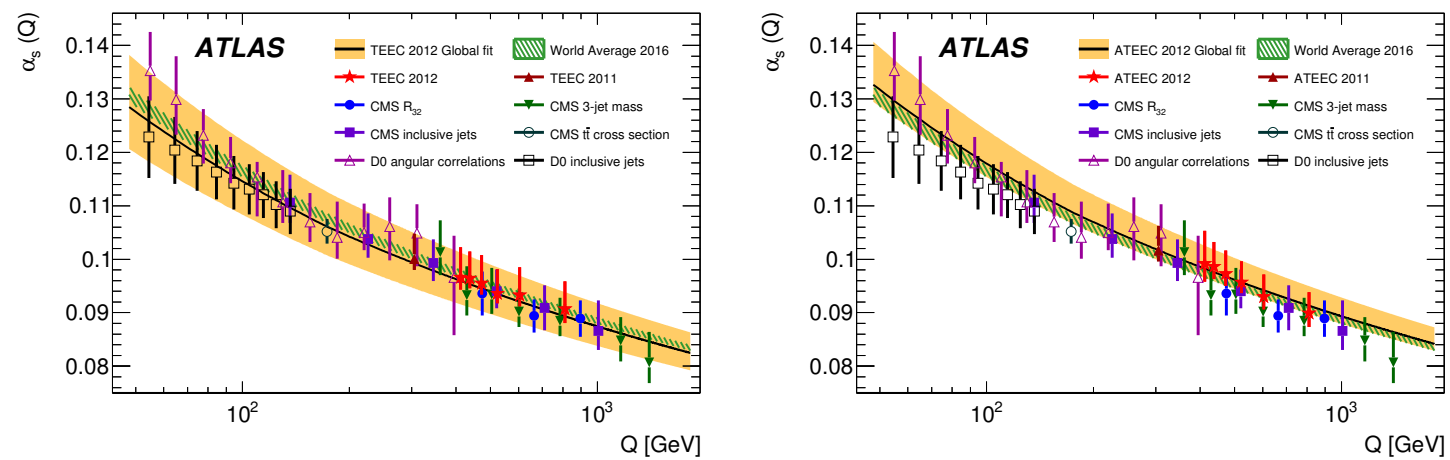

Figure 5: Comparison of the values of $\alpha_{s}(Q)$ obtained from fits to the TEEC (left) and ATEEC (right) functions at the energy scales given by $\left\langle H_{T 2}\right\rangle / 2$ (red star points) with the uncertainty band from the global fit (orange solid band) and the 2016 world average (green hatched band). Determinations from other experiments are also shown as data points. [7] 


\section{References}

[1] ATLAS Collaboration, 2008 JINST 3 S08003.

[2] ATLAS Collaboration, JHEP02(2015)153.

[3] ATLAS Collaboration, JHEP 09 (2017) 020.

[4] ATLAS Collaboration, ATLAS-CONF-2017-048.

[5] James Currie, E.W.N. Glover, Joao Pires, Phys. Rev. Lett. 118, 072002 (2017); James Currie, E.W.N. Glover, Aude Gehrmann-De Ridder, Thomas Gehrmann, Alexander Huss, Joao Pires, arXiv: 1704.00923.

[6] ATLAS Collaboration, Physics Letters B 750 (2015) 427-447.

[7] ATLAS Collaboration, arXiv:1707.02562. Submitted to EPJC. 